Schmerz 2017 $\cdot 31: 404$

DOI 10.1007/s00482-017-0223-0

Online publiziert: 2. Juni 2017

(c) Deutsche Schmerzgesellschaft e.V. Published by Springer Medizin Verlag $\mathrm{GmbH}$ - all rights reserved 2017

CrossMark

F. Kühne ${ }^{1} \cdot$ C. Meinders ${ }^{2} \cdot$ H. Mohr ${ }^{3} \cdot$ K. Hafenbrack ${ }^{4} \cdot$ K. Kieseritzky ${ }^{5}$.

C. Rosenberger ${ }^{3} \cdot$ M. Härter ${ }^{3} \cdot$ F. Schulz-Kindermann ${ }^{3} \cdot$ R. Klinger ${ }^{6} \cdot$ A. Y. Nestoriuc ${ }^{7}$

${ }^{1}$ Abteilung für Klinische Psychologie und Psychotherapie, Universität Potsdam, Potsdam, Deutschland

${ }^{2}$ Arbeitsbereich Klinische Psychologie und Psychotherapie, Institut für Psychologie, Universität Hamburg, Hamburg, Deutschland

${ }^{3}$ Institut und Poliklinik für Medizinische Psychologie, Universitätsklinikum Hamburg-Eppendorf (UKE), Hamburg, Deutschland

${ }^{4}$ Psychologisch-psychotherapeutische Praxis, Hamburg, Deutschland

${ }^{5}$ Psychologischer Dienst, Krankenhaus St. Joseph-Stift Bremen, Bremen, Deutschland

${ }^{6}$ Klinik und Poliklinik für Anästhesiologie, Bereich Schmerzmedizin und Schmerzpsychologie, Universitätsklinikum Hamburg-Eppendorf (UKE), Hamburg, Deutschland

${ }^{7}$ Institut und Poliklinik für Psychosomatische Medizin und Psychotherapie, Universitätsklinikum, Hamburg-Eppendorf (UKE), Hamburg, Deutschland

\title{
Psychologische Interventionen zur Schmerzreduktion
}

dies in Rechnung, bleibt die Vergleichbarkeit beider Werte erhalten.

Die Evidenzsynthese erfolgte narrativ in Text- und Tabellenform, d. h. in Form einer strukturierten Zusammenfassung und Diskussion von Studien [1].

Um unsere Arbeit $\mathrm{zu}$ fokussieren, hätten wir eine weitergehende Gegenüberstellung wie auch eine Überprüfung von Zitaten und Übersetzungen selbstverständlich vorgenommen, wenn wir den Hinweis dazu vor Publikation erhalten hätten.

Der Leserbrief fokussiert in weiten Teilen auf das Gutachterwesen, weshalb wir ausschließlich auf die inhaltlichen Punkte im Zusammenhang mit unserer Arbeit eingehen. Untersucht wurden schmerzpsychologische Interventionen, wie beschrieben definiert als psychologische Interventionen, deren primäres Ziel die Schmerzreduktion war.

Die extrahierten Zielgrößen, wie Lebensqualität oder Depressivität, ergaben sich aus den in den Primärstudien untersuchten Hauptoutcomes und nicht aus der Suchstrategie.

Zur Einschätzung der methodischen Qualität der Primärstudien konnte ein Kriterium des von Johannsen und Kollegen [2] gebildeten Scores nicht berücksichtigt werden, da die eingeschlossenen Primärstudien keine metaanalytische Zusammenfassung erlaubten. Stellt man

\section{Korrespondenzadresse}

Dipl.-Psych. Dr. phil. F. Kühne

Abteilung für Klinische Psychologie und Psychotherapie, Universität Potsdam Karl-Liebknecht-Str. 24-25, 14476 Potsdam, Deutschland

dr.franziska.kuehne@uni-potsdam.de

Interessenkonflikt. F. Kühne, C. Meinders, H. Mohr, K. Hafenbrack, K. Kieseritzky, C. Rosenberger, M. Härter, F. Schulz-Kindermann, R. Klinger und A.Y. Nestoriuc geben an, dass kein Interessenkonflikt besteht.

\section{Literatur}

1. Higgins JPT, Green S (Hrsg) (2011) Cochrane handbook for systematic reviews of interventions. Version 5.1.0 [updated March 2011]. The Cochrane Collaboration, London (Available from www. handbook.cochrane.org)
2. Johannsen M, Farver I, Beck N, Zachariae R (2013) The efficacy of psychosocial intervention for pain in breast cancer patients and survivors: a systematic review and meta-analysis. Breast Cancer Res Treat 138:675-690 